\title{
Optimizing X-Ray Imaging Using Plant Mediated Gold Nanoparticles as Contrast Agent: A Review
}

\author{
Gbadebo A. Isola, Margaret K. Akinloye, Yekinni K. Sanusi, Paul S. Ayanlola*, Gabriel A. Alamu \\ Department of Pure and Applied Physics, Ladoke Akintola University of Technology, Ogbomoso, Nigeria \\ Corresponding author*
}

\begin{abstract}
X-ray imaging has been a clinical practice for more than half a century, and the hydrophilic iodinated-based molecules are universally and continuously employed as contrast agents. However, a renewed interest in nanoparticles-based contrast agents has emerged with the promise of detailed imaging, and potential for therapeutic applications. Preclinical and clinical applications of nanoparticles are identified for a broad spectrum of imaging applications, with commentaries on the future promise of these materials. However, these nanomaterials contrast agents are not without issues, as the method of synthesizing the nanomaterials limits their applications as clinical contrast agents. The physical and chemical methods of synthesizing nanoparticles have been proven successful but not biocompatible due to the use of toxic chemicals, but, with the biocompatibility of biological substances such as microorganisms and plants, nanoparticles can be synthesized for applications in x-ray imaging.
\end{abstract}

Key words: Contrast agents, Gold nanoparticles, Optimizing, Plant, X-ray Imaging

\section{INTRODUCTION}

$\mathrm{T}$ he use of ionizing radiation and the utilization of contrast agents in medical imaging has allowed for a more effective and timely diagnosis and treatment of patients. However, due to some limitations of the clinically approved iodinated contrast agents, nanoparticles, most especially, gold nanoparticles (AuNPs) have been studied and are reported to be candid replacements for clinical use as x-ray contrast agents. The nanoparticles promise improved abilities for invivo applications and potentially enhanced targeting efficiencies through longer engineered circulation times, designed clearance pathways and binding capacities [1]. Hence, this paper takes a concise review on the concept of $\mathrm{x}$ ray imaging and the currently employed contrast agents, contribution of nanotechnology to medicine, different methods of synthesizing AuNPs and also call for the investigation and utilization of plants in synthesizing AuNPs for the purpose of $\mathrm{x}$-ray imaging.

\section{CONCEPT OF X-RAY IMAGING}

In medical imaging, images of internal body structures require the transfer of energy from a source, which interacts with the human body and finally to an appropriate receptor for display [2], [3]. The source of energy employed may be different, but, the energy must be able to penetrate the human body, deposit certain portion of its energy in human tissue and interact with internal structures in a manner that will create image information (Figure 1). The use of ionizing radiation (IR) in medical imaging to accurately and timely diagnose health problems, has allowed for a more effective treatment of patients. This makes medical images, most especially x-ray imaging a window to the human body [2]. Reports also have it that over $90 \%$ of the population total exposure from manmade sources of IR is from diagnostic use of x-rays, as the number of patients undergoing nuclear medicine and radiotherapy is much lower than the number undergoing diagnostic procedures [4].

The purpose of x-ray imaging is to obtain images of internal tissues and organs suitable for diagnostic and therapeutic investigations. However, some anatomical features can be so similar in physical characteristics to their surroundings that they cannot be radiographically distinguished from the surrounding tissues and fluids. More so, radiation exposure through medical imaging is a variable factor and often has an effect on image quality. Improving the quality of x-ray images always implies increase in the radiation dose to the patient, which in turn increases the radiation risk (Figure 1). Thus, the increasing availability of imaging modalities that utilize $\mathrm{x}$-rays owing to the technological advancement must be justified and optimized in order to keep radiation as low as reasonably achievable (ALARA). Hence, the purpose of $\mathrm{x}$-ray imaging is not just to deliver the perfect image but one that is diagnostically adequate for the specific health problem, which is the essence of optimization, as this is necessary so as to balance image quality with radiation dose without compromising the diagnostic information being sought [5], [6].

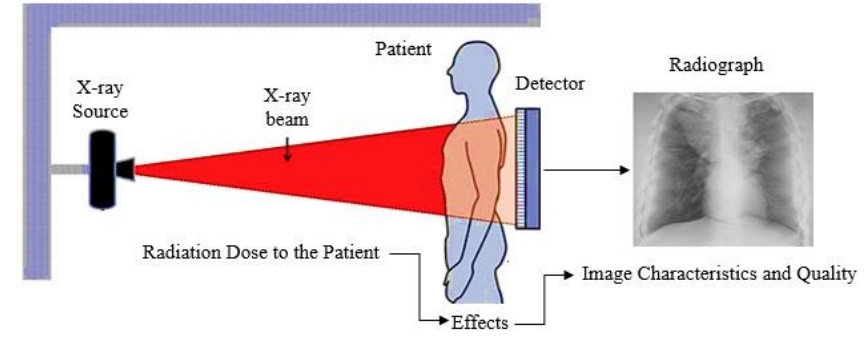

Fig 1. Concept of medical imaging (adapted from [2])

\section{X-RAY İMAGING CONTRAST AGENTS}

In order to assist medical practitioners most especially radiographer and radiologist, to visualize abnormalities on 
radiographic image and to achieve efficient and high resolution images, contrast agents (CAs) are utilized in different modalities of x-ray imaging [7], [8]. The CAs are required purposely to enhance the visibility of internal structures such as tissues and organs, since soft biological tissues lack sufficient contrast [9]. The CAs are required to improve the contrast between tissues that attenuate $\mathrm{x}$-rays similarly [10]. Some of the X-ray imaging modalities that employ CA are projection radiography, computed tomography, and fluoroscopy. Contrast agents are of great importance in $\mathrm{x}$-ray imaging because they greatly increase the sensitivity of an imaging technique allowing the diagnosis of previously undetectable diseases. The CAs absorb external Xrays, resulting in decreased exposure on the $\mathrm{x}$-ray detector, they also make certain structures or tissues in the body appear different on the image than they would without the contrast material. However, the CA to be used must be of high atomic number $(Z)$ to provide an adequate change in attenuation, it must also be economical, non-toxic and possess a viscosity suitable for either injection or ingestion [11].

Iodine- and barium-based molecules are the commonly used $\mathrm{CA}$ in $\mathrm{x}$-ray imaging. The iodinated contrast agents (ICAs) may be administered intravenously, while the barium molecules may be taken orally. These agents produce contrast with respect to soft tissue because of their atomic number and densities, which increase the attenuation of the $x$-ray. The significance of their atomic numbers iodine $(Z=53)$ and barium $(Z=56)$ is that, they cause the k-absorption edge (iodine $=33 \mathrm{keV}$ and barium $=37 \mathrm{keV}$ ) to be located at a very favourable energy relative to the typical $x$-ray energy spectrum. Interest in these agents has declined not only because of their atomic number but because of their low kshell binding energy [12], [13].

This is because, the ICAs are small molecules that are based on a tri-iodinated benzene ring and the iodinated rings are typically functionalized with amides and alcohol groups to provide water solubility. The ICAs can be described in terms of viscosity; osmolality (high, low, or isotonic); ionicity (ionic or non-ionic) and the number of benzene rings (monomer or dimer), all of which yield different properties in terms of toxicity and clearance from the blood stream. Viscosity is the ability of the formulation to flow freely. This is strongly influenced by the concentration of the $\mathrm{CA}$ and the temperature of the solution [14]. It has been reported that highly viscous agents limit the rate of injection and might cause pain at the site of injection [15]. Osmolality is the molality of an ideal solution that would exert the same osmotic pressure as the solution being considered. It is important to decrease the viscosity and osmolality of CA so as to achieve a quality radiographic image suitable for diagnostic information.

The ionic form has high osmolality in the order of 1500 mosm $\mathrm{kg}^{-1}$. Examples of ionic ICAs include diatrizoate (under the name Hypaque), metrizoate (Isopaque), iothalamate (Conray), and ioxaglate (Hexabrix; Mallinckrodt). The ionic form was found to be the source of chemotoxicity, such as renal toxicity causing nephrosis, vasodilation, pulmonary hypertension and many more [10], [16]. Pain, sensation of heat at the site of injection and metallic taste may also be experienced. A patient with history of allergy to iodine increases the risk for allergic reactions such as asthma. The risk of kidney damage from ionic form of $\mathrm{CA}$ is higher in patients with poor renal function, diabetes, dehydration, congestive heart failure, or age above seventy years [17].

The non-ionic form has more advantage over the ionic type as they are less toxic and safer to use because they have a lower tendency to interact with cell membranes, peptides and other biological structures [17] - [19]. The non-ionic monomer form has low osmolality in the range of $600-700$ mosm kg-1 . Examples are iohexol (under the name Omnipaque; GE Healthcare), iopromide (Ultravist; Bayer Healthcare), and iopamidol (Isovue; Bracco Imaging). However, because the osmolality was still found to be excessive, non-ionic dimer was introduced. The dimer form has osmolality around 300 mosm $\mathrm{kg}^{-1}$ and are thus isotonic [14]. An example of the dimer form is iodixanol (Visipaque; GE Healthcare).

Non-ionic dimers show lower osmolalities than the non-ionic monomers, which reduces pain and sensation of heat at the site of injection [19], decreases the incidence of contrast induced nephrotoxicity (CIN), which is a common condition in patients with pre-existing renal impairment, cardiovascular complications such as heart rate and blood pressure irregularities and osmotic diuresis [14], [19]. Nevertheless, dimers tend to exhibit higher viscosities than monomers [18], which is something of concern. Additionally, slow excretion rates have been associated in preclinical studies with upregulation of markers for renal injury [20]. This situation usually limits the use of dimers in CT and angiography imaging, as these techniques require high injection rates [15]. Hence, a balance between osmolality and viscosity values must be found for each ICA.

Despite the efforts made to improve the physicochemical properties and molecular structure of existing CAs, the clinically approved ICAs suffer from rapid renal clearance from circulation after intravenous injection [20]. Given this rapid clearance, large amounts of contrast agents are often required for prolonged or multiple $\mathrm{x}$-ray imaging, and injecting large volumes of ICA may trigger serious adverse reactions [21] - [24] and increase the chance of severe renal impairment. It is thus evident that the currently employed CAs provide limited contrast, which can lead to inadequate diagnosis [9], [25]. The CAs also suffer from nonspecific biodistribution, short circulation half-lives, a requirement for catheterization. Moreover, one of the biggest concerns about the utilization of ICAs is related to the fact that they have a greater density than plasma, resulting in extreme hyperosmolarity [26], [27]. It is in view of all these limitations that researchers are looking for alternative means to develop a novel contrast agent that will be superior in terms of atomic number and absorption coefficient and perform better at 
diagnostic energy level, that nanotechnology is making a significant impact to the field of medical imaging.

\section{CONTRIBUTION OF NANOTECHNOLOGY TO MEDICINE}

The concept of nanotechnology presented by Richard Feynman in a presentation titled was described as a process of manipulating and controlling matter on a miniature scale, creating nanomaterials atom by atom or molecule by molecule [28]. Ever since then, nanotechnology has developed into an interdisciplinary field that involves the study and application of natural sciences and engineering to modify and develop tools at the nanoscale [27]. The field focuses on the design, development, characterization and the use of materials with at least one dimension less than $100 \mathrm{~nm}$. Nanomaterials are broadly divided into various categories depending on their morphology, size and chemical properties.

The tremendous growth of the field is turning the present world into a "nanoworld" as the miniaturization of particles have been found applicable in a myriad of fields and disciplines. It is within this context that, nanomedicine, an indisputable research area has evolved. The integration of nanomaterials into medicine has led to the development of diagnostic devices, contrast agents, analytical tools, physical therapy applications, drug delivery vehicles and many more (Figure 2). Of utmost interest to researchers, is the call for the introduction of nanoparticles (NPs) as contrast agents in X-ray imaging to overcome the limitations of the clinically approved contrast agents and to aid in proper evaluation and understanding of disease diagnosis and treatment, at the same time justify and optimize the radiation doses that a patient receives as part of medical diagnosis and therapy [25].

In order to determine whether or not the NPs are suitable for imaging and other medical applications, an accurate evaluation of the properties must be performed before safe administration into the body. This, however depends on the ability to synthesize particles with different physicochemical properties such as atomic number, size, shape, morphology, concentration and functional group, as these are the primary factors governing how nanomaterial agents interact with biological systems [1], [29]. Recently, metal NPs have gained pre-clinical interest for use as contrast agents in imaging, as some elements possess higher atomic number than iodine and barium, and these elements can further provide improved $\mathrm{x}$ ray attenuation at diagnostic energy ranges due to the higher k-edge energy levels compared to iodinated agents.

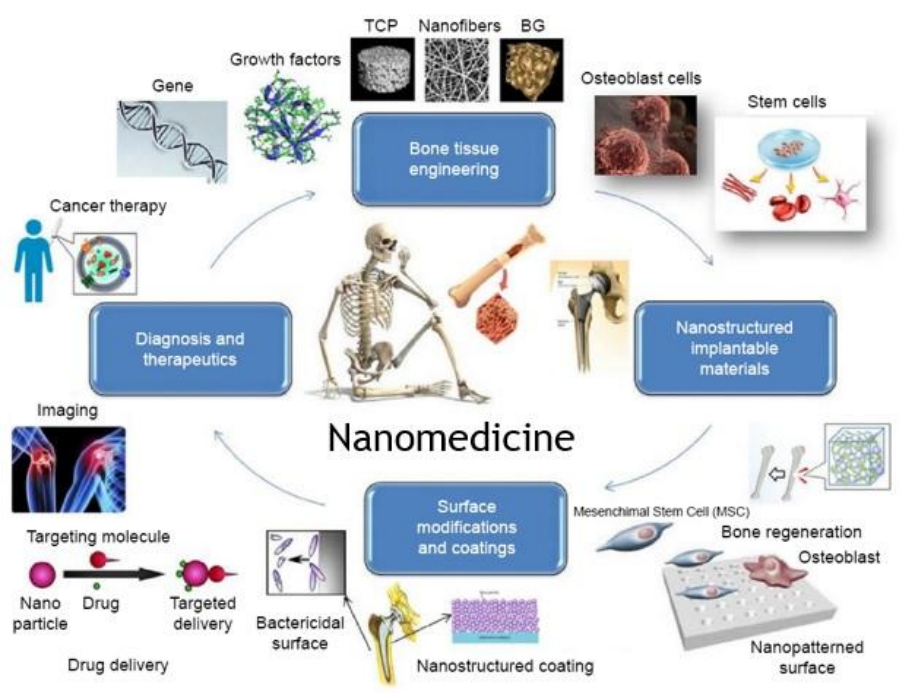

Fig 2. Potential applications of nanomaterials in medicine (adapted from [30])

Of the variety of elements with higher $Z$, for example, lanthanide $(Z=57)$, gadolinium $(Z=64)$, ytterbium $(Z=70)$, tantalum $(Z=73)$, gold $(Z=79)$ and bismuth $(Z=83)$, gold incorporated nanoparticles (AuNPs) have been studied extensively for use as a novel x-ray contrast agent. This is because, AuNPs possess excellent x-ray attenuation properties, unique optical properties, high electron conductivity and good biocompatibility, that make them applicable in many areas of life. More so, the synthesis of AuNPs allows for control of the physicochemical properties. The advantages of AuNPs in comparison to other formulated NPs-based imaging agents include their simple surface modification for achieving colloidal stability and enabling active targeting. Given the favourable x-ray attenuation results of $\mathrm{Au}$, the toxicity of AuNPs has been evaluated in both in vitro and in vivo studies, showing that they can be modified with other molecules employed in biomedical applications.

Hainfeld et al. [18] reported the production of $1.9 \mathrm{~nm}$ AuNPs from Nanoprobes Inc. USA, and intravenously injected into mice at a volume of $0.01 \mathrm{mLg}^{-1}$. The mice were thereafter imaged using a clinical mammography unit and comparison of pre- and post- injection x-ray images revealed that the agent strongly opacified the blood vessels of the animal. No toxicity was observed from haematology and histology 11 days and 30 days after injection. Encouraged by the results, several studies have been conducted using different reducing and capping agents to further modify the properties of AuNPs purposely for further biomedical applications [31], [32]. This has made AuNPs gain attention and are being considered as a suitable candidate for $\mathrm{x}$-ray imaging to replace iodinated agents [33], [34].

\section{SYNTHESIS OF GOLD NANOPARTICLES}

The methods that have been developed to produced NPs, have been categorized into two approaches, namely: top-down or bottom-up [28], [35] - [38]. In the top-down approach, the synthesis follows a destructive process, in which the 
preparation begins with the bulk material of interest, and later undergoes size reduction via either the physical or chemical process to produce NPs. This approach does not have good control of the particle size and structure. In the bottom-up approach, the synthesis follows a building up process, in which the NPs are prepared from the atomic or molecular level (Figure 3).

The physical methods are based on the energy transfer that occurs in a material when irradiated by either ionizing or nonionizing radiation, which triggers the reduction reaction that leads to the nucleation of metallic particles. The physical methods include gamma irradiation, laser ablation, microwave irradiation, hydrothermal, ball milling, thermal evaporation, lithography and high energy irradiation and many more [39] [41]. Physical methods may involve the use of hazardous materials as reducing and stabilizing agents to prevent agglomeration, which may not be environmentally friendly. Also, the technologies involved are expensive and require high energy and space [42] - [44].

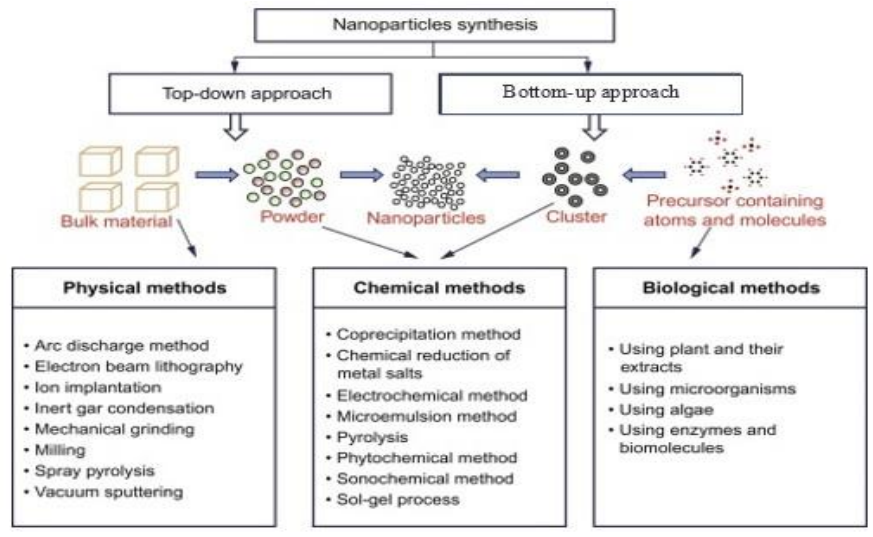

Fig 3. Different methods of synthesizing nanoparticles (adapted from [41]

The chemical routes are the most common and require strong reducing agents to initiate the synthetic process and promote NPs nucleation. Most of the chemical routes include chemical precipitation, sol-gel method, solution-evaporation method, photochemical reduction, electrochemical, templating, and sonochemical techniques and many more [45] - [48]. Chemically prepared NPs have been reported to be inappropriate for medical applications due to the use of toxic solvents, contamination from precursor chemicals and production of hazardous chemicals binding on their surface [48].

In synthesising AuNPs, $\mathrm{Au}$ precursor has been found to be unstable, therefore a suitable reducing agent is required to prevent aggregation. The synthesis method also plays a vital role in controlling the size and surface area of nanomaterials. It is evident that both chemical and physical routes have been successful in producing well-defined AuNPs, but these processes require the use of toxic chemicals, high energy and space, and often involve the use of expensive equipment and technologies. Therefore, the chemically and physically prepared AuNPs are not useful in medical and biological applications [49]. The considerations of some of the challenges often encountered in both the chemical and physical methods of synthesizing NPs, have resulted in numerous studies that have suggested new routes of synthesising AuNPs. This new approach employs biological means as reduction and protection agents.

The biological methods involve the use of microorganisms such as actinomycetes, bacteria, fungus, virus, algae, yeast and plants. Biological methods have been suggested as clean, reliable, biocompatible, benign and eco-friendly alternatives to chemical and physical methods [38], [50], [51]. Thus, biological synthesis has proven to be a better method due to slower kinetics, better manipulation and control over crystal growth and stabilization. However, there is still a considerable level of research that needs to be undertaken to assess and elucidate the differences in size, morphology and other features between NPs synthesized from biological sources. In view of the difficulties faced in microbe assisted synthesis [52], plant mediated synthesis is developing because of the ease of handling and controlling the size and shape of the NPs.

The three main steps involved in the synthesis of NPs based on green chemistry perspectives are the selection of a biocompatible and nontoxic solvent medium; choosing of environmentally favourable reducing agents; and nontoxic substances for stabilization of the NPs. In the approach, $\mathrm{HAuCl}_{4}$ will be employed as a precursor and reduced by utilizing agents obtained from biological substances. The synthesis procedure involves mixing of metallic salt with extracts of the biological substances (Figure 4) for definite amount of time under varied reaction conditions such as $\mathrm{pH}$, incubation time and temperature to obtain NPs of specific shapes and sizes. The formation of NPs is thereafter indicated by a change in colour of the reaction mixture. After which the synthesised solution will be characterized using different spectroscopy and microscopy thechniques to determine the nature, structure and composition of solution and truly confirm that synthesised solution is of nanoscale.
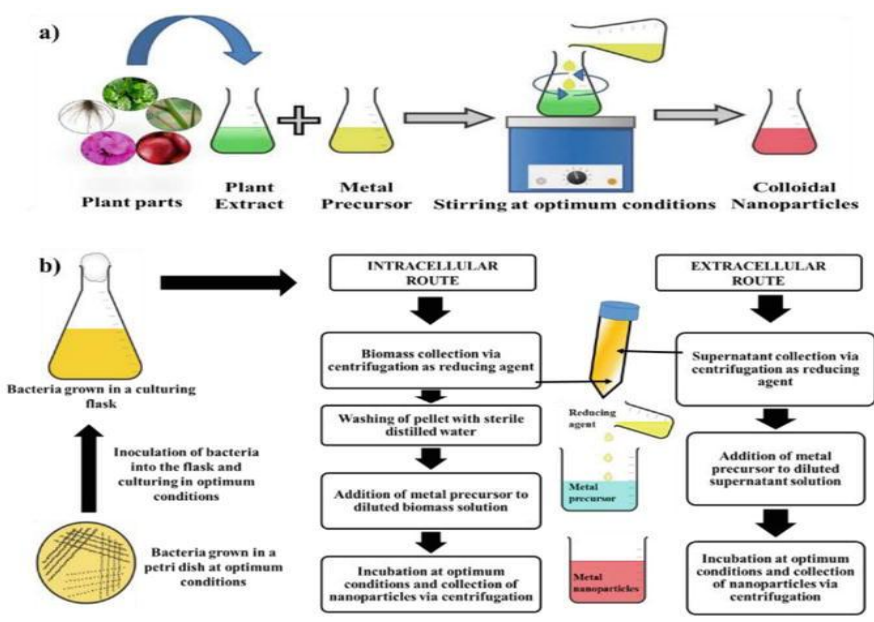

Fig 4. Biological methods (a) plant based (b) microbial synthesis of nanoparticles (adapted from [53]) 
Plants mediated synthesis of NPs have been reported to have various advantages in comparison to the microorganisms due to their availability, faster, being relatively easy, low cost, and non-toxic behaviour [54]. It has long been known that plants have the potential to hyper-accumulate and biologically reduce metallic ions [55]. Furthermore, using plants and plant materials in synthesizing NPs is advantageous over other biological processes since it eliminates the elaborate process of maintaining bacterial and fungal cultures. Also plants do not need multi-step procedures such as isolation, culture preparation and maintenance [50], [56] - [59], therefore the process can be easily scaled up for large scale production of beneficial NPs [60]. Thus, plants have been proven to be excellent reducing and stabilizing agents for the biosynthesis of AuNPs in a clean, reliable and biofriendly way [61].

Reviewing through literature, it can be established that, AuNPs have been successfully synthesized using different plants and most of which have been investigated and reported for different biological applications such as antiparasitic [62], [63], anticancer [64], [65], antioxidant [66], [67], antibacterial [67] and still counting. However, the potential application of plant mediated AuNPs as x-ray contrast agents has not been reported in literature. Thus, with diverse bioactive components present in plants, the synthesis of AuNPs using plants extract can reposition the utilization of nanoparticles as contrast agent in X-ray imaging over other methods and further optimize the use of ionizing radiation in medical imaging.

\section{FUTURE PROSPECTIVE}

It is evident that nanomedicine is developing at a rapid rate, while human understanding of the physicochemical properties, efficiency and toxicity of $\mathrm{x}$-ray contrast agents is also improving. This understanding can be used to develop a novel NP-based contrast agent that will provide higher resolution at lower concentration and, thus reduce the adverse effects and costs associated with imaging procedures and at the same time enhance patient welfare during diagnoses. The design and evaluation of NP-based x-ray contrast agents have been proven effective to replace the clinically approved iodinated $\mathrm{x}$ ray contrast agents and, thus improve $x$-ray imaging. Of the several elements that possess higher atomic number than iodine and barium, gold became one of the first prospects to prepare iodine-free NP based x-ray contrast agents because it possesses a high biotolerability and a low cytotoxicity, these characteristics make it suitable for use for biological applications. Several studies have also demonstrated that AuNPs exhibit longer blood circulation times, lower toxicity and, most importantly, enhanced x-ray attenuation capabilities.

Although significant progress has been made in preparing NP based x-ray contrast agents from elements with higher atomic number and better x-ray attenuation properties than iodine, still, it is necessary to conduct more research regarding the effect of the size and distribution of the NPs on x-ray absorption capabilities. It is clear that both the chemical and physical routes of synthesizing AuNPs are successful in producing well-defined AuNPs, some of which are undergoing series of trials for use as X-ray imaging contrast agents. More so, different plants have been used to synthesis AuNPs for diverse purposes, with little or no study reporting the imaging applications. However, the synthesis of AuNPs using plant extracts has advantages over the other methods as it is safe, eco-friendly and simple to use. The approach can also be used to produce large quantities of NPs. Hence, a detailed study is needed to give a clear understanding of the green synthesis of AuNPs using biomolecules present in plants which will be valuable to improve AuNPs properties and further enhance the imaging application.

\section{CONCLUSION}

The use of ionizing radiation in medical imaging has come to stay, and iodinated based molecules are continuously employed as contrast agents in x-ray imaging. As useful as the existing contrast agents are, they pose challenges to human health, thereby limiting their imaging applications. With the introduction of nanoparticles into medicine, a renewed interest in nanoparticles-based contrast agents has emerged with the promise of detailed imaging, and potential for therapeutic applications. Gold nanoparticles are one of the most promising nanomaterials for various applications especially for use as contrast agents in $\mathrm{x}$-ray imaging. It is evident that lots of scientific studies have been carried out on synthesising AuNPs through physical and chemical means, yet a lot still needs to be done regarding the biological means most especially the use of plants based nanomaterials. Plants have been proven to possess high efficiency as reducing and stabilizing agents for the synthesis of controlled materials in terms of sizes, shapes, structures, and other features. More so, as the paradigm of environmental protection is swinging toward green environment, plant based nanoparticles really requires to be fully explored in view of their being environmentally friendly. Thus, the present review calls for the utilization of plants in the synthesis of AuNPs and since preliminary investigations support the potential for AuNPs in biomedical applications, efforts should be made to investigate the suitability of plant synthesized AuNPs as contrast agents in X-ray imaging and thereby validate the biosafety of the nanomaterials.

\section{REFERENCES}

[1] Cole LE, Ross RD, Tilley JMR, Vargo-Gogola T, Roeder RK. Gold nanoparticles as contrast agents in X-ray imaging and Computed Tomography, Nanomedicine (Lond.), 2015; 10(2): 321 - 341. doi: 10.2217/nnm.14.171

[2] Sprawls P. Physical Principles of Medical Imaging, Second Edition, Medical Physics Publishing Madison, Wisconsin, 1993.

[3] Kasban H, El-Bendary MAM, Salama DH. A comparative Study of Medical Imaging Techniques. International Journal of Information Science and Intelligent System 2015; 4 (2): 37-58.

[4] Martin A, Harbison S, Beach K, Cole P. An Introduction to Radiation Protection, $7^{\text {th }}$ Edition CRC Press Taylor \& Francis Group, 2019.

[5] International Commission on Radiation Protection (ICRP). The 2007 Recommendations of the International Commission on Radiological Protection. ICRP Pub. No. 103. Ann ICRP. 2007; $37: 1-332$ 
[6] International Atomic Energy Agency (IAEA). Optimizing radiation dose for medical image quality . IAEA 2020.

[7] Cormode DP, Skajaa T, Fayad ZA, Mulder WJM. Nanotechnology in medical imaging: Probe design and applications. Arteriosclerosis Thrombosis and Vascular Biology 2009; 29(7): 992-1000. doi: 10.1161/atvbaha.108.165506

[8] Loudos G, Kagadis GC, Psimadas D. Current status and future perspectives of in vivo small animal imaging using radiolabelled nanoparticles. European Journal of Radioliogy 2011; 78 (2): $287-$ 295. doi: 10.1016/j.ejrad.2010.06.025

[9] Chien CC, Chen HH, Lai SF, Wu KC, Cai X et al. Gold nanoparticles as high-resolution X-ray imaging contrast agents for the analysis of tumor-related micro-vasculature. Journal of Nanobiotechnology 2012; 10(1): 1-12.

[10] Loveless VS. Adjunct agents used in X-ray imaging. In: Diagnostic Imaging for Pharmacists (ed. B. Templar Smith and K.D. Weatherman) 43-64. Washington, DC: AphA, 2012.

[11] Flower MA. Webb's Physics of Medical Imaging Second Edition, CRC Press 2012

[12] National Institute of Standards and Technology (NIST), Physical Measurement Laboratory. X-ray transition energy: K-edge table. USA, 1996.

[13] Hoheisel M. Review of medical imaging with emphasis on X-ray detectors. Nuclear Instruments and Methods in Physics Research Section A 2006; 563(1): 215-224. doi: 10.1016/j.nima.2006.01.123

[14] Krause W, Schneider P. Chemistry of X-ray contrast agents. In: Krause W. (eds) Contrast Agents II. Topics in current Chemistry 222 Springer 2002. doi: 10.1007/3-540-46009-8_4

[15] Newington IM, Humphries G, Lasbistes N, Morisson-Iveson V, Nairne $\mathbf{J}$ et al. The synthesis and evaluation of trimeric X-ray contrast agents. Tetrahedron Letters 2011; 52 (24): 3065-3067. doi: 10.1016/j.tetlet.2011.04.010

[16] Yu SB, Watson AD. Metal-based x-ray contrast media. Chemical Reviews 1999; 99:2353-77.doi: 10.1021/cr980441p

[17] Reilly RM Medical Imaging for Health professionals: Technologies and Clinical Applications, First Edition, Wiley and Sons, Inc 2019.

[18] Hainfeld JF, Slatkin DN, Focella T M, Smilowitz HM. Gold nanoparticles: A new X-ray contrast agent. British Journal of Radiology 2006; 79(939): 248-253. doi: 10.1259/bjr/13169882

[19] Lusic H, Grinstaff MW. X-ray computed tomography contrast agents. Chem Rev., 2013; 113(3): 1641-1666. Doi: $10.1021 \% 2 \mathrm{Fcr} 200358 \mathrm{~s}$

[20] Wynn DG, Humphries G, Morisson-Iveson V, Nairne J, Newington IM et al. The synthesis and evaluation of unsymmetrical dimeric X-ray contrast agents. Tetrahedron Letters 2011; 52 (24): 3068-3071. doi: 10.1016/j.tetlet.2011.04.009

[21] Rand D, Ortiz V, Liu Y, Derdak Z, Wands JR et al. Nanomaterials for X-ray imaging: gold nanoparticle enhancement of X-ray scatter imaging of hepatocellular carcinoma. Nano Letters 2011; 11: 2678-2683. doi: 10.1021/n1200858y

[22] Liu Z, Li Z, Liu J, Gu S, Yuan Q et al. Long-circulating $\mathrm{Er}^{3+}$ doped $\mathrm{Yb}_{2} \mathrm{O}_{3}$ up-conversion nanoparticle as an in vivo X-ray $\mathrm{CT}$ imaging contrast agent. Biomaterials 2012; 33 (28): 6748-6757. doi: 10.1016/j.biomaterials.2012.06.033

[23] Lee N, Choi SH, Hyeon T. Nano-sized CT contrast agents. Advanced Materials 2013; 25(19):2641-2660. doi: 10.1002/adma.201300081

[24] He W, Ai K, Lu L. Nanoparticulate X-ray CT contrast agents. Science China Chemistry 2015; 58:753-760. doi: 10.1007/s11426015-5351-8

[25] Gundogdu O, Nirgianaki E, Che Ismail E, Jenneson PM, Bradley DA. Benchtop phase-contrast X-ray imaging. Applied Radiation and Isotope 2007; 65 (12): 1337-1344. doi: 10.1016/j.apradiso.2007.07.009

[26] Todd B. Iodine-based contrast agents. Geriatr Nurs., 1987; 8(6): 341-348.

[27] De La Veg JC, Häfeli UO. Utilization of nanoparticles as X-ray contrast agents for diagnostic imaging applications. Contrast
Media and Molecular Imaging 2015; 10: 81-95. doi: $10.1002 / \mathrm{cmmi} .1613$

[28] Khan I, Saeed K, Khan I. Nanoparticles: Properties, applications and toxicities. Arabian Journal of Chemistry 2019; 12(7): 908 931. doi: 10.1016/j.arabjc.2017.05.011

[29] Rosen JE, Yoffe S, Meerasa A, Verma M, Gu FX. Nanotechnology and Diagnostic Imaging: New Advances in Contrast Agent Technology. Journal of Nanomedicine and Nanotechnology 2011; 2(5): 1-12. doi: 10.4172/2157-7439.1000115

[30] Mazaheri M, Eslahi N, Ordikhani F, Tamjid E, Simchi A. Nanomedicine applications in orthopedic medicine: state of the art. International Journal of Nanomedicine 2015; 10: 6039-6054. doi: 10.2147/ijn.s7373

[31] Kim D, Park S, Lee JH, Jeong YY,Jon S. Antibiofouling polymercoated gold nanoparticles as a contrast agent for in vivo $\mathrm{X}$-ray computed tomography imaging. Journal of American Chemical Society 2007; 129(24): 7661-7665. doi: 10.1021/ja071471p

[32] Boote E, Fent G, Kattumuri V, Casteel S, Katti K et al. Gold nanoparticle contrast in a phantom and juvenile swine: models for molecular imaging of human organs using x-ray computed tomography. Academic Radiology 2010; 17(4): 410-417. doi: 10.1016/j.acra.2010.01.006

[33] Giljohann DA, Seferos DS, Daniel WL Massich MD, Patel PC et al. Gold nanoparticles for biology and medicine. Angewandte Chemie (International ed. in English) 2010; 49:3280-3294. doi: $10.1002 \% 2$ Fanie. 200904359

[34] Lamanna G, Kueny-Stotz M, Mamlouk-Chaouachi H et al. Dendronized iron oxide nanoparticles for multimodal imaging. Biomaterials., 2011; 32(13): 8562-8573. doi: 10.1016/j.biomaterials.2011.07.026

[35] Sepeur S, Laryea N, Goedicke S, Grob F. Nanotechnology: Technical Basics and Applications; Vincentz Network GmbH \& Co: Hannover, Germany 2019. doi:10.1515/9783748602347

[36] Nielson R, Kaehr B., Shear JB. Micro-replication and design of biological architectures using dynamic-mask multi-photon lithography. Small, 2009; 5(1): 120-125. doi: 10.1002/smll.200801084

[37] Birnbaum AJ, Pique A. Laser induced extra-planar propulsion for three dimensional microfabrication. Applied Physics Letters 2011; 98 (13): 134101-134106. doi: 10.1063/1.3567763

[38] Shah M, Fawcett D, Sharma S, Tripathy SK, Poinern GEJ. Green Synthesis of Metallic Nanoparticles via Biological Entities. Materials, 2015; 29(8): 7278 - 7308. doi: 10.3390/ma8115377

[39] Mafune F, Kohno J, Takeda Y, Kondow TJ. Dissociation and aggregation of gold nanoparticles under laser irradiation. The Journal of Physical Chemistry B 2001; 105: 9050-9056.

[40] Zhang G, Wang DJ. Fabrication of heterogeneous binary arrays of nanoparticles via colloidal lithography. Journal of the American Chemical Society 2008; 130(17): 5616-5617. doi: $10.1021 / \mathrm{ja} 710771 \mathrm{j}$

[41] Devatha CP, Thalla AK. Green Synthesis of Nanomaterials in Synthesis of Inorganic Nanomaterials Advances and Key Technologies, a volume in Micro and Nano Technologies 2018; 7: 169 - 184. doi: 10.1016/B978-0-08-101975-7.00007-5

[42] Gade A, Bonde P, Ingle A, Marcato P, Duran N, Rai M. Exploitation of Aspergillus niger for synthesis of silver nanoparticles. Journal of Biobased Materials and Bioenergy 2008; 2(3): 243-247. doi: 10.1166/jbmb.2008.401

[43] Ai J, Biazar E, Jafarpour M, Montazeri M, Majdi A et al. Nanotoxicology and nanoparticle safety in biomedical designs. International Jounal of Nanomedicine. 2011; 6: 1117-1127. doi: 10.2147\%2FIJN.S16603

[44] Wei X, Luo M, Li W, Yang L, Liang X et al. Synthesis of silver nanoparticles by solar irradiation of cell-free Bacillus amyloliquefaciens extracts and AgNO3. Bioresource Technology 2012; 103(1), 273-278. doi: 10.1016/j.biortech.2011.09.118

[45] Chen W, Cai W, Zhang L, Wang G, Zhang L. Sonochemical processes and formation of gold nanoparticles within pores of mesoporous silica. Journal of Colloid and Interface Science 2001; 238(2): 291-29. doi: 10.1006/jcis.2001.7525 
[46] Rodríguez-Sanchez L, Blanco MC, Lopez-Quintela MA. Electrochemical synthesis of silver nanoparticles. The Journal of Physical Chemistry B 2000; 104(41): 9683-9688. doi: 10.1021/jp001761r

[47] Eustis S, Hsu HY, El-Sayed MA. Gold nanoparticle formation from photochemical reduction of $\mathrm{Au}^{3+}$ by continuous excitation in colloidal solutions: A proposed molecular mechanism. The Journal of Physical Chemistry B 2005; 109(11): 4811-4815. doi: $10.1021 /$ jp0441588

[48] Starowiicz M, Stypula B, Banas J. Electrochemical synthesis of silver nanoparticles. Electrochemistry Commununications 2006; 8(2): 227-230. doi: 10.1016/j.elecom.2005.11.018

[49] Shankar SS, Rai A, Ahmad A, Sastry MJ. Rapid synthesis of Au, $\mathrm{Ag}$, and bimetallic $\mathrm{Au}$ core- $\mathrm{Ag}$ shell nanoparticles using Neem (Azadirachta indica) leaf broth. Journal of Colloid and Interface Science 2004; 275(2): 496- 502. doi: 10.1016/j.jcis.2004.03.003

[50] Vigneshwaran N, Ashtaputre NM, Varadarajan PV, Nachane RP, Paralikar KM et al. Biological synthesis of silver nanoparticles using the fungus Aspergillus flavus. Materials Letter 2007; 61(6): 1413-1418. doi: 10.1016/j.matlet.2006.07.042

[51] Zhang W, Yong D, Huang J, Yu J, Liu S et al. Fabrication of Polymer-Gadolinium (III) Complex Nanomicelle from Poly(ethylene glycol)-Polysuccinimide Conjugate and Diethylenetriaminetetraacetic Acid-Gadolinium as Magnetic Resonance Imaging Contrast Agents. Journal of Applied Polymer Science 2011; 120(5): 2596-2605. doi: 10.1002/app.33464

[52] Nath D, Banerjee P. Green Nanotechnology-A New Hope for Medical Biology. Environmental Toxicology and Pharmacology 2013; 36(3): 997-1014. doi: 10.1016/j.etap.2013.09.00

[53] Moholkar D.N., Havaldar D.V., Potadar R.S. and Pawar K.D. (2020). Optimization of Biogenic Synthesis of Colloidal Metal Nanoparticles. In Colloids- Types, Preparation and Applications, Infotech; 1 - 29 doi: 10.5772/intechopen.9485

[54] Kale A, Bao Y, Zhou Z, Prevelige PE, Gupta A. Directed selfassembly of CdS quantum dots on bacteriophage P22 coat protein templates. Nanotechnology 2013; 24(4) 045603. Doi:10.1088/0957-4484/24/4/045603

[55] Swami A, Selvakannan PR, Pasricha R, Sastry M. One-step synthesis of ordered two dimensional assemblies of silver nanoparticles by the spontaneous reduction of silver ions by pentadecylphenol Langmuir monolayers. The Journal of Physical Chemistry B 2004; 108, 19269-19275. doi: 10.1021/jp0465581

[56] Bar H, Bhui DK, Sahoo GP, Sarkar P, de Sankar P et al. Green synthesis of silver nanoparticles using latex of Jatropha curcas.
Colloids and Surfaces A: Physicochemical and Engineering Aspects 2009; 339(1): 134-139. doi: 10.1016/j.colsurfa.2009.02.008

[57] Jha AK, Prasad K, Kulkarni AR. Plant system: Nature's nanofactory. Colloids and Surfurces B: Biointerfaces 2009; 73(2): 219-223. Doi: b10.1016/j.colsurfb.2009.05.018

[58] Thakkar KN, Mhatre SS, Parikh RY. Biological synthesis of metallic nanoparticles. Nanomedicine: Nanotechnology, Biology and Medice 2010; 6(2): 257-262. doi: 10.1016/j.nano.2009.07.002

[59] Yasmin A, Ramesh K, Rajeshkumar S. Optimization and stabilization of gold nanoparticles by using herbal plant extract with microwave heating. Nano Convergence, 2014; 1(12): 1- 7. doi: 10.1186/s40580-014-0012-8

[60] Ahmed S, Ikram S. Synthesis of Gold Nanoparticles using Plant Extract: An Overview. Nano Research \& Applications 2015; 1 (1): $1-6$.

[61] Soni N, Prakash S. Green nanoparticles for mosquito control. The Scientific World Journal 2014; 1. doi: 10.1155/2014/496362

[62] Rahul S, Chandrashekhar P, Hemant B, Bipinchandra S, Mouray E et al. In vitro antiparasitic activity of microbial pigments and their combination with phytosynthesized metal nanoparticles. Parasitol Int., 2015; 64(5): 353-356. doi: 10.1016/j.parint.2015.05.004

[63] Patil MP, Kim GD. Eco-friendly approach for nanoparticles synthesis and mechanism behind antibacterial activity of silver and anticancer activity of gold nanoparticles. Applied Microbiology and Biotechnology 2017; 101:79-92. doi 10.1007/s00253-0168012-8

[64] Patil MP, Ngabire D, Thi HHP, Kim MD, Kim GD. Eco-friendly synthesis of gold nanoparticles and evaluation of their cytotoxic activity on cancer cells. Journal of Cluster Science 2017; 28:119132. doi: 10.1007/s10876-016-1051-6

[65] Lakshmana A, Umamaheswari C, Nagarajan NS. A facile phytomediated synthesis of gold nanoparticles using aqueous extract of Momordica cochinchinensis rhizome and their biological activities. Journal of Nanoscience and Technology 2016. 2:76-80.

[66] Vijayan R, Joseph S, Mathew B. Eco-friendly synthesis of silver and gold nanoparticles with enhanced antimicrobial, antioxidant, and catalytic activities. IET Nanobiotechnology, 2018; 12(6): 850 856. doi: 10.1049/iet-nbt.2017.0311

[67] Rad MR, Kazemian H, Yazdani F, Monfared MRZ, Rahdar H et al. Antibacterial activity of gold nanoparticles conjugated by aminoglycosides against A. baumannii isolates from burn patients. Recent Patents on Anti-infective Drug Discovover 2018; 13(3)256264 doi: 10.2174/1574891X13666180828115543. 